\title{
Multiple Criteria-Based Real-Time Decision Making by Autonomous City Vehicles
}

\author{
A. Furda and L. Vlacic \\ Intelligent Control Systems Laboratory (ICSL), \\ Institute of Integrated and Intelligent Systems, \\ Griffith University, Brisbane, QLD 4111, Australia \\ (e-mail:a.furda@griffith.edu.au; l.vlacic@griffith.edu.au)
}

\begin{abstract}
This paper addresses the topic of real-time decision making for autonomous city vehicles, i.e. the autonomous vehicles' ability to make appropriate driving decisions in city road traffic situations. After decomposing the problem into two consecutive decision making stages, and giving a short overview about previous work, the paper explains how Multiple Criteria Decision Making (MCDM) can be used in the process of selecting the most appropriate driving maneuver.
\end{abstract}

Keywords: autonomous city vehicles, real-time decision making, multiple criteria decision making

\section{INTRODUCTION}

One of the most crucial aspects which, in the near future, will make autonomous driving in city traffic possible, is the autonomous vehicles' ability to make safe and appropriate driving decisions in any city traffic situation. Without reliable real-time decision making, human drivers cannot be safely replaced by computer-based vehicle control and decision making systems.

This paper addresses the question on enabling driverless city vehicles to identify the most appropriate driving maneuver to be performed under the given road traffic circumstances, since so far developed solutions are not sufficient for safe autonomous driving in the complex realworld city traffic conditions.

The remainder of this paper is structured as follows. Subsection 1.1 gives an overview of the state of research, and subsection 1.2 presents our so far work on the development of the autonomous vehicle's control and decision making system. Section 2 explains how Multiple Criteria Decision Making (MCDM) can be applied for autonomous driving, including a discussion about benefits and drawbacks, and experimental tests. Section 3 concludes this presentation.

\subsection{State of Research}

The so far published material about autonomous city vehicles did not reveal any evidence that the problem of realtime decision making for such vehicles has already been addressed using decision theory methods, and focusing on a generic solution for the real-world (non-simplified) city traffic conditions. A large variety of solutions, for example using automata theory, decision trees, heuristic approaches, or priority queues have been published, however, these solutions were developed for very specific applications in simplified conditions, and, according to their developers, would not work in real-world city traffic conditions.

The major recent event demonstrating the state of the art driverless city vehicle technology was the DARPA Urban Challenge 2007 (DARPA (2006)), a driverless vehicle race in a simulated urban environment. However, although all vehicles competed in the same traffic environment, and had therefore identical decision making requirements, a direct comparison between their approaches is difficult due to varying terminology used in presenting similar ideas. For instance, the vehicle "Boss" had a "behavioral system" consisting of "subcomponents" (Urmson et al. (2008)), while the vehicle "Junior " had "navigation modules" executing "behaviors" or "actions" (Montemerlo et al. (2008)). Nevertheless, the following is an attempt to give an overview based on a unified terminology, as interpreted by the authors of this paper.

The winning vehicle "Boss" was able to perform three main driving maneuvers: lane following, intersection handling and driving in a parking area. Its decision making capabilities consisted of choosing and executing one of these three driving maneuvers depending on the vehicle's location (Urmson et al., 2008).

The vehicle "Junior" achieved the 2nd place with a decision making approach based on a finite automaton with 13 states (Montemerlo et al., 2008). Since each automaton state maneuvered the vehicle in a specific traffic situation (e.g. stop sign, cross intersection, U-turn), each state can be seen as part of a driving maneuver.

Similar to "Junior ", "Odin's" (3rd place) decision making approach was based on a finite automaton, however hierarchically structured. Driving maneuvers for parking areas, road following, and intersections were chosen based on a so-called "winner-takes-all arbitration method" (Bacha et al., 2008). 
The vehicle "Little Ben's" decision making approach consisted of choosing between the driving maneuvers lane following, U-turns, intersections and parking areas, depending on the vehicle's location (Bohren et al., 2008), similar to the winning vehicle "Boss".

The vehicle "Knight Rider" used a combination of reasoning and finite automata for decision making. The last choice between executable driving maneuver alternatives was made based on fixed priorities (Patz et al., 2008).

The vehicle "Skynet" performed four driving maneuvers: driving on roads, crossing intersections, driving in parking zones and handling road blockages. Its decision making approach was based on heuristic decision rules defined in the form of a decision tree (Miller et al., 2008).

The vehicle "AnnieWAY's" decision making was based on a hierarchical finite automaton, each state representing a driving maneuver for a specific situation (e.g. intersection handling) (Kammel et al., 2008).

The vehicle "Caroline" was able to perform five driving maneuvers: follow way points, stay in lane, avoid obstacles, stay on road, stay in (parking) zone. Its decision making approach was based on heuristics implemented as weighted votes (Rauskolb et al., 2008).

Although these vehicles impressively demonstrated that it was possible to successfully complete DARPA Urban Challenge test track, it is important to note that this race took place in a simplified environment, and not in realworld traffic. The developed decision making solutions for the DARPA Urban Challenge were focused on fulfilling the race requirements with the overall goal to win the race, and not on enabling driverless vehicles to safely cope with real-world city traffic. For example, the developers of "Knight Rider" allowed their vehicle to perform illegal U-turns in order to save time (Patz et al. (2008)), while team "Talos" released safety constraints by decreasing safety distances around obstacles, ignoring traffic lanes, "neglecting " the part of the vehicle behind its rear axle, or skipping checkpoints (Leonard et al. (2008)).

The developers of the winning vehicle "Boss" noticed that their traffic representation was not sufficient to make intelligent driving decisions compared to human drivers, and that their development approach was "ad hoc" (Urmson et al., 2008), driven by testing. As they state, the developed concepts "are able to reliably complete these tests but are not robust to a varied world" (Urmson et al., 2008).

Driverless city vehicles for civilian, non-military applications are not likely to gain public acceptance unless they prove to be safer than conventional human-driven vehicles. Therefore, the decision making subsystem plays a crucial role toward reaching this goal.

\subsection{Previous Work}

In previous work, we have addressed and developed solutions for the following:

- Control System Architecture consisting of four functional subsystems (Figure 1):

- Perception Subsystem: collects, manages and processes available information about the vehicle's road traffic environment, and provides it through the World Model to the Real-Time De- cision Making \& Driving Maneuver Control, and Driving Maneuvers.

- Real-Time Decision Making \& Driving Maneuver Control: makes driving decisions based on the information provided by the Perception Subsystem. This software subsystem is the main focus of this paper and further explained below.

- Driving Maneuvers: closed-loop control algorithms, able to maneuver the vehicle in a specific traffic situation. All driving maneuvers are structured in a common way, their operational behaviors are modeled as deterministic finite automata. Details have been published in (Furda and Vlacic (2009)).

- Vehicle Interface: low-level vehicle control (e.g. speed and steering angle).

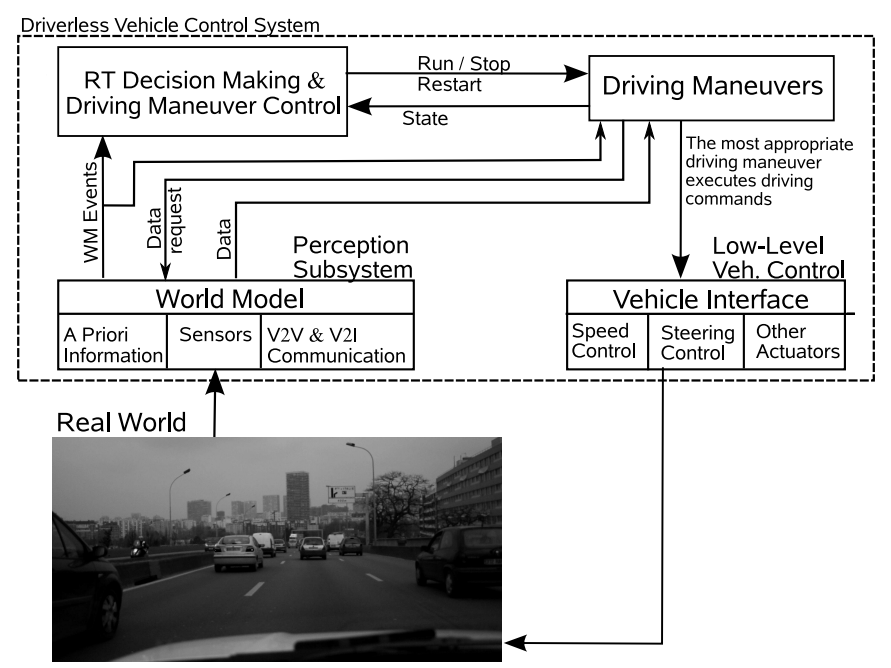

Fig. 1. Simplified view of the driverless vehicle control software architecture and the flow of data.

Furthermore, we have decomposed the problem of RealTime Decision Making into two consecutive stages (Figure 2):

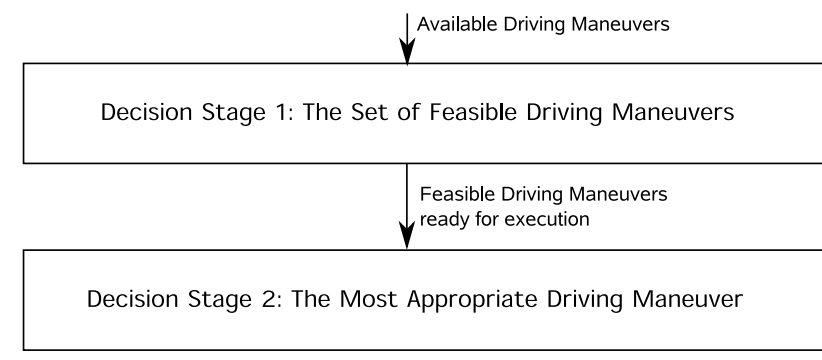

Fig. 2. The decision making process consisting of two consecutive stages.

- Decision Stage 1: Selects the set of feasible driving maneuvers, i.e. those driving maneuvers which can be safely performed, and which conform to the road traffic rules. The approach used in this first stage is based on discrete events and a Petri Net model, which enables not only the analysis and simulation, but also the formal algorithm verification of this safetycritical decision making stage. Details about this first decision stage have been published in (Furda and Vlacic (2009)). 
- Decision Stage 2: Selects and activates the most appropriate driving maneuver from the set of feasible ones. Since the set of feasible driving maneuvers only contains those maneuvers which can be safely performed in the specific road traffic situation, this stage is not safety centric. However, it focuses on a variety of other important objectives, such as maximizing the efficiency, comfort, or minimizing travel times. This second stage is the main topic of this paper and is further elaborated in section 2 , below.

\section{MULTIPLE CRITERIA-BASED DECISION MAKING (MCDM)}

\subsection{Selecting the Most Appropriate Driving Maneuver}

The goal of the second decision making stage (Figure 2) is to select and execute the most appropriate alternative from those driving maneuvers which have been determined to be feasible in the current traffic situation.

Each of the feasible driving maneuvers offers multiple execution alternatives, which can be selected through discrete driving maneuver parameters. For instance, the overtaking maneuver could be performed at low or high speed, in distant or close proximity to the front vehicle, and on the right or left hand side. In order to select the most appropriate driving maneuver, and for it the most appropriate execution alternative, we apply Multiple Criteria Decision Making (MCDM) as follows.

Objectives: we define a hierarchy of objectives starting from a main, most general driving objective, which is then further successively broken down into more specific and therefore more operational objectives on lower hierarchy levels. Eventually, the bottom level of the objective hierarchy contains only objectives $o b j_{j}$ which are fully operational and which are measurable through their attributes (Figure 3).

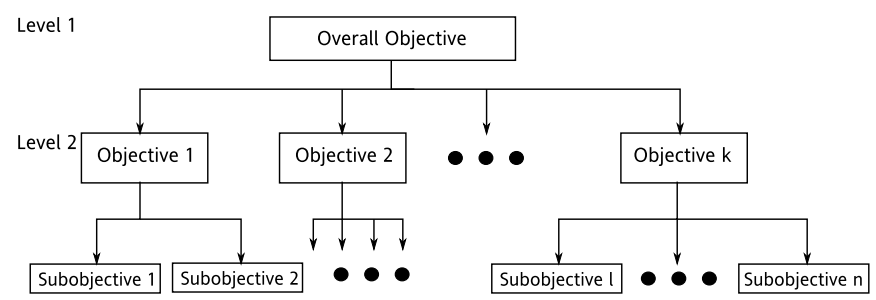

Fig. 3. Hierarchy of objectives (Chankong and Haimes (1983)).

The most general objective for autonomous driving is to safely reach the specified destination. More precisely, this objective is broken down into a lower hierarchy level containing more specific objectives, which specify how to achieve the objective of the higher level. Thus, we define the following objective hierarchy consisting of four $(k=4)$ level 2 objectives:

- Drive to destination safely $=: o b j^{\text {Level1 }}$

- Stay within road boundaries $=: o b j_{1}^{\text {Level } 2}$ keep distance to right boundary $:=a t t r_{1}$ keep distance to left boundary $:=a t t r_{2}$ - Keep safety distances $=: o b j_{2}^{\text {Level } 2}$ keep distance to front vehicle $:=\operatorname{attr}_{3}$ keep distance to moving obstacles $:=a^{a t t} r_{4}$ keep distance to static obstacles $:=$ attr $_{5}$

Do not collide $=: o b j_{3}^{\text {Level } 2}$

keep minimum distance to obstacles $:=a_{t} t_{6}$ drive around obstacles $:=a^{t} t r_{7}$ avoid sudden braking $:=a t r_{8}$ avoid quick lane changes $:=a t t r_{9}$

- Minimize waiting time $=: o b j_{4}^{\text {Level2 }}$ maintain minimum speed $:=$ attr $_{10}$ avoid stops $:=a t t r_{11}$

Attributes: a set of measurable attributes

$$
\left\{a t t r_{1}, a t t r_{2}, . ., a t t r_{p}\right\}, p \in \mathbb{N} \text { ( } \mathbb{N}=\text { set of natural numbers) }
$$

is assigned to each objective on the lowest hierarchy level (in our example it is level 2 with $p=11$ ). An attribute is a property of a specific objective. In order to define various levels of importance, weights may be assigned to each attribute.

Alternatives: in the context of our application, decision alternatives correspond to the execution of driving maneuvers. Therefore, in a first step, we regard each element of the set of driving maneuvers $\left\{M^{1}, M^{2}, . ., M^{n}\right\}(n \in \mathbb{N})$ to be an element of the set of alternatives $A$ :

$$
A=\left\{M^{1}, M^{2}, . ., M^{n}\right\}
$$

However, each driving maneuver $M^{m}(1 \leq m \leq n)$ offers one or multiple execution alternatives by specifying discrete $^{1}$ parameter values (e.g. fast/slow, close/far, etc.). The driving maneuver parameters correspond in MCDM terms to decision variables, where each alternative is respresented by a decision variable vector.

We obtain:

$$
\begin{gathered}
M^{1}=\left\{M_{1}^{1}, M_{2}^{1}, . ., M_{j}^{1}\right\} \\
M^{2}=\left\{M_{1}^{2}, M_{2}^{2}, . ., M_{k}^{2}\right\} \\
\vdots \\
M^{n}=\left\{M_{1}^{n}, M_{2}^{n}, . ., M_{l}^{n}\right\},
\end{gathered}
$$

where $n$ denotes the number of driving maneuvers, and $j$, $k, l$ the number of execution alternatives for the maneuvers $M^{1}, M^{2}$, and $M^{n}$ respectively.

Therefore, the set of alternatives $A$ contains all execution alternatives of all $n$ driving maneuvers:

$$
A=\bigcup_{m=1}^{n} M^{m}=\left\{M_{1}^{1}, M_{2}^{1}, . ., M_{j}^{1}, M_{1}^{2}, . ., M_{k}^{2}, . ., M_{1}^{n}, . . M_{l}^{n}\right\}
$$

For the sake of readability, we denote all alternatives as:

$$
A=\left\{a_{1}, a_{2}, . ., a_{q}\right\},(q=j+k+. .+l)
$$

Utility Functions: utility functions $f_{1}\left(a_{i}\right), \ldots, f_{p}\left(a_{i}\right)$ specify the level of achievement of an objective by an alternative $a_{i} \in A(i \in[1, q])$ with respect to each of the $p$ attributes.

For each attribute $\operatorname{attr}_{i}(i \in[1, p])$, we define a utility function $f_{a t r_{i}}=f_{i}$ :

$$
f_{i}: A \rightarrow[0,1]
$$

\footnotetext{
1 In order to reduce the computational costs, we discretize the otherwise continuous parameter values of driving maneuvers.
} 
Consequently, defining utility functions $f_{i}$ for all alternatives $a_{1}, a_{2}, . ., a_{q}$ and all attributes $\operatorname{attr}_{i}(i \in[1, p])$ results in the following decision matrix:

$$
\begin{array}{c||c|c|c|c}
a_{i} & \text { attr } & \text { attr } & \ldots & \text { attr } \\
\hline \hline a_{1} & f_{1}\left(a_{1}\right) & f_{2}\left(a_{1}\right) & \ldots & f_{p}\left(a_{1}\right) \\
a_{2} & f_{1}\left(a_{2}\right) & f_{2}\left(a_{2}\right) & \ldots & f_{p}\left(a_{2}\right) \\
\vdots & \vdots & \vdots & \vdots & \vdots \\
a_{q} & f_{1}\left(a_{q}\right) & f_{2}\left(a_{q}\right) & \ldots & f_{p}\left(a_{q}\right)
\end{array}
$$

The remaining problem is to select the best among the feasible alternatives. A variety of MCDM methods can be applied in order to solve this problem, such as dominance methods, satisficing methods, sequential elimination methods, or scoring methods (Yoon and Hwang (1995)). In the following example we choose a widely used scoring method, the Simple Additive Weighting Method, in which the value $V\left(a_{i}\right)$ of an alternative $a_{i}$ is calculated by multiplying the utility function values with the attribute weights and then summing the products over all attributes (see equation 1) (Yoon and Hwang (1995)). The alternative with the highest value is then chosen.

\subsection{Example}

In this example we assume the traffic situation shown in Figure 4. The vehicle on the left side is a driverless vehicle, passing a stopped vehicle.

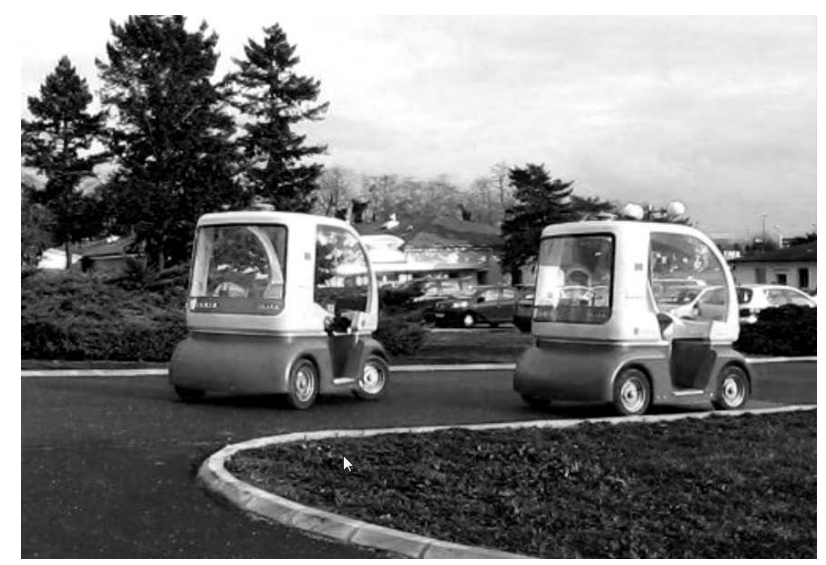

Fig. 4. Driverless vehicle (left) passing a stopped vehicle (right) (right-hand side driving).

For this situation, without oncoming traffic, the first decision making stage determined the following two driving maneuvers as feasible: Passing the stopped vehicle, or Stop\&Go (i.e. waiting behind the temporarily stopped vehicle).

For the sake of simplicity, we assume that only the following few execution alternatives for the two driving maneuvers are possible:

- Passing maneuver $M^{1}$ :

- $a_{1}:=$ speed $=$ slow, lateral distance $=$ small

- $a_{2}:=$ speed $=$ slow, lateral distance $=$ large

- $a_{3}:=$ speed $=$ fast, lateral distance $=$ small

- $a_{4}:=$ speed $=$ fast, lateral distance $=$ large

- Stop\&Go maneuver $M^{2}$ :

- $a_{5}:=$ distance to front vehicle $=$ small

- $a_{6}:=$ distance to front vehicle=large
Consequently, the set of feasible alternatives is:

$$
A=\left\{a_{1}, a_{2}, . ., a_{6}\right\}
$$

The utility functions $f_{i}(A)$ evaluate the achievement level of each attribute $i$ for each of the 6 alternatives. In order to allow comparisons between the levels of achievement of different objectives, the values of the utility functions $f_{i}$ are scaled to a common measurement scale, the interval of real numbers between 0 and 1 . We define:

$$
f_{i} \in[0,1] \subset \mathbb{R}
$$

where the value 1 denotes the optimal achievement of an objective, while 0 denotes that the objective is not achieved at all.

We define the utility functions as follows. Each of the 6 alternatives are rated regarding on how well they fulfill the driving objectives on the lowest hierarchy level. We rate the alternatives on a scale from 0 to 1 , where:

- 1 denotes optimal fulfillment of the objective,

- 0.75 denotes good fulfillment,

- 0.5 denotes indifference,

- 0.25 denotes bad fulfillment,

- 0 denotes unsatisfactory fulfillment.

In our example, the utility function values are assigned based on heuristics reflecting the preferences of a human driver, as listed in Table 1.

For calculating the best solution, we choose in this example the Simple Additive Weighting Method (Yoon and Hwang (1995)). We define the value of an alternative $a_{i}$ as follows:

$$
V\left(a_{i}\right):=\sum_{j=1}^{p} w_{j} f_{j}\left(a_{i}\right),
$$

where $p$ denotes the number of attributes.

Each attribute is assigned a weight $w_{j}$, which reflects its importance. For autonomous driving, the importance of various objectives changes depending on the road conditions. For example, on a wide boulevard at higher speed, the attribute "attr 8 : avoid sudden braking" is more important than the attribute "attr ${ }_{1}$ : keep distance to right boundary". However, in a residential area, the opposite might be the case. Consequently, instead of defining invariable attribute weights, this method offers the possibility to adapt the attribute weights, and therefore the decision preferences, according to the current traffic environment. In our example, we define the attribute weights as listed in Table 1.

Using the utility functions and attribute weights as listed in Table 1, we calculate the value of each alternative:

$$
\begin{aligned}
V\left(a_{1}\right)= & \sum_{j=1}^{11} w_{j} f_{j}\left(a_{1}\right) \\
= & 1 * 1+1 * 0.5+2 * 0.5+1 * 0.5+1 * 0.25 \\
& +1 * 0.25+1 * 1+3 * 0.5+2 * 0.75+2 * 0.75 \\
& +2 * 1=11.0
\end{aligned}
$$


Table 1. Heuristic definition of utility functions $f_{i}: A \rightarrow[0,1]$ for the 6 alternatives $a_{1}, . ., a_{6}$ and 11 attributes $a t t r_{1}, . ., a t t r_{11}$. Weights indicate the level of importance. The column $V\left(a_{i}\right)$ lists the calculated values for each alternative $a_{i}$.

\begin{tabular}{|c|c|c|c|c|c|c|c|c|c|c|c|c|}
\hline$a_{i}$ & $\operatorname{attr}_{1}$ & $a_{t t r}$ & $\operatorname{attr}_{3}$ & $\operatorname{attr}_{4}$ & $\operatorname{attr}_{5}$ & $\operatorname{attr}_{6}$ & $\operatorname{attr}_{7}$ & $\operatorname{attr}_{8}$ & $\operatorname{attr}_{9}$ & $a t t r_{10}$ & $a_{t t r}$ & $V\left(a_{i}\right)$ \\
\hline$a_{1}$ & 1 & 0.5 & 0.5 & 0.5 & 0.25 & 0.25 & 1 & 0.5 & 0.75 & 0.75 & 1 & 11 \\
\hline$a_{2}$ & 1 & 0.25 & 0.5 & 0.5 & 1 & 1 & 1 & 0.5 & 0.75 & 0.75 & 1 & 12.25 \\
\hline$a_{3}$ & 1 & 0.5 & 0.5 & 0.5 & 0.25 & 0.25 & 1 & 1 & 0.25 & 1 & 1 & 12 \\
\hline$a_{4}$ & 1 & 0.25 & 0.5 & 0.5 & 1 & 1 & 1 & 1 & 0.25 & 1 & 1 & 13.25 \\
\hline$a_{5}$ & 0.5 & 0.5 & 0.25 & 0.5 & 0.25 & 0.25 & 0 & 0 & 1 & 0 & 0 & 4.5 \\
\hline$a_{6}$ & 0.5 & 0.5 & 1 & 0.5 & 0.75 & 1 & 0 & 0.25 & 1 & 0 & 0 & 8 \\
\hline Weight & $w_{1}=1$ & $w_{2}=1$ & $w_{3}=2$ & $w_{4}=1$ & $w_{5}=1$ & $w_{6}=1$ & $w_{7}=1$ & $w_{8}=3$ & $w_{9}=2$ & $w_{10}=2$ & $w_{11}=2$ & \\
\hline
\end{tabular}

$V\left(a_{2}\right)=12.25 ; \quad V\left(a_{3}\right)=12.0 ; \quad V\left(a_{4}\right)=13.25 ; \quad V\left(a_{5}\right)=4.5$

$V\left(a_{6}\right)=8.0$

The highest value $\max _{1 \leq i \leq 6} V\left(a_{i}\right)=13.25$ is achieved by alternative $a_{4}$ (passing at fast speed with a large lateral distance to the stopped vehicle). Therefore, this alternative is chosen for execution.

\subsection{Discussion}

Multicriteria Decision Making methods are established mathematical tools which are widely applied in a large variety of engineering and science fields with complex decision problems (Stadler (1988), White (1976)). For our problem of autonomous driving, MCDM offers a variety of benefits:

- The hierarchy of objectives allows a systematic and complete specification of goals to be achieved by the vehicle.

- The utility functions can be defined heuristically to reflect the choices of a human driver, or, alternatively, learning algorithms can be applied.

- MCDM allows the integration and evaluation of a very large number of driving alternatives.

- Decision flexibility can be achieved by defining the set of attribute weights depending on the road conditions.

- Additional objectives, attributes, and alternatives can be added without the need of major changes.

However, since the method is highly based on heuristics (i.e. heuristic definition of objectives, utility functions, and attributes), if MCDM alone is used (without the first decision making stage (Figure 2)), it is difficult the ensure that all made decisions will always lead to safe driving. We have addressed this problem, by ensuring that the MCDM process only selects the most appropriate driving maneuver from the set of feasible driving alternatives, which is the outcome of the first decision stage (Figure 2 ).

\subsection{Experimental Test Results}

In order to demonstrate the decision making functionality, all other components, most of all the Perception Subsystem, but also Driving Maneuvers, are required.

The Decision Stage 1 outcomes have already been demonstrated in both 3D simulation (Figure 5) (Boisse et al.
(2007)) and in on-road experiments with Cycab vehicles (Figure 4) (Furda and Vlacic (2009)).

So far, the second decision making stage has been tested in the $3 \mathrm{D}$ simulation. Figure 6 shows the 3D simulation environment and the decision making graphical user interface. In the shown traffic scenario, the autonomous vehicle (left) approaches a stopped vehicle. The first decision making stage determines three driving maneuvers as feasible: overtaking (passing), platooning (stop\&go), and emergency stop. The second, MCDM-based decision making stage correctly assesses the overtaking maneuver as the most appropriate one, which is then performed by the vehicle control software (Figure 1).

In the shown simulation, we have also obtained correct decision results for other situations, such as approaching an intersection, or avoiding collisions with pedestrians and static obstacles.

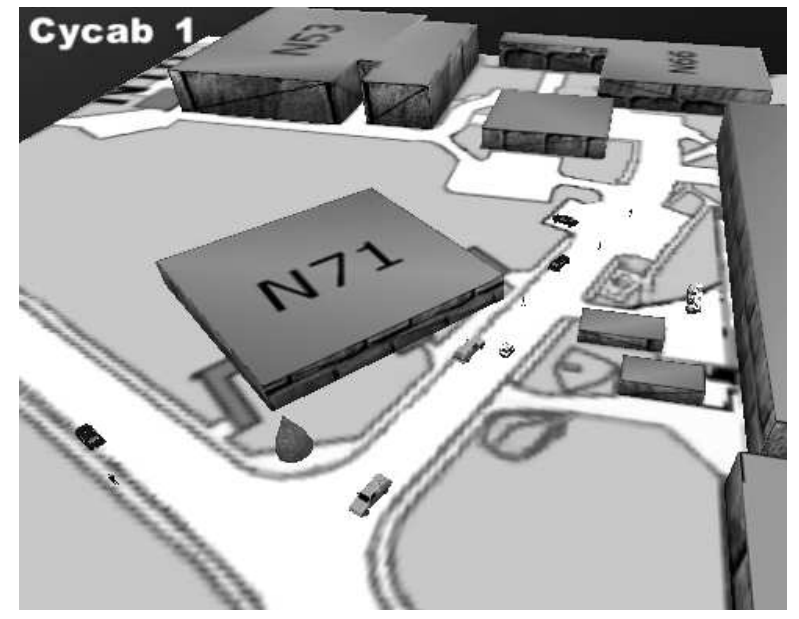

Fig. 5. The 3D simulation environment is a model of the test environment for the real driverless vehicle at our Nathan Campus (Griffith University).

\section{CONCLUSION}

This paper has addressed the problem of Real-Time Decision Making for autonomous city vehicles. After decomposing the problem into two consecutive decision making stages, and demonstrating a solution for the first decision making stage, we have designed and developed the MCDM model, which is then applied in the second decision making stage. 


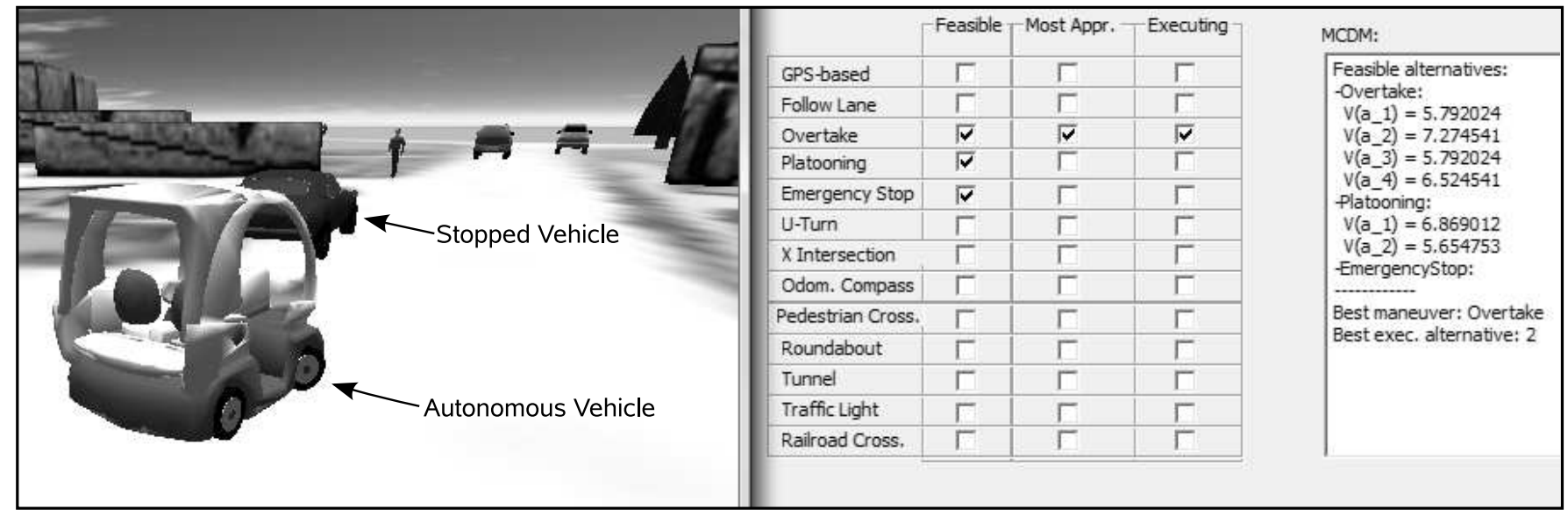

Fig. 6. 3D simulation environment and the decision making graphical user interface of the autonomous vehicle control software (edited screenshot, left-hand side driving).

A step-by-step explanation, accompanied by an example, showed how MCDM can be applied, in order to decide about the most appropriate driving maneuver.

Compared to so far existing solutions, the application of MCDM offers a variety of benefits with respect to the problem specification, decision flexibility, and scalability.

We have demonstrated the first stage of our decision making approach in both a $3 \mathrm{D}$ simulation and real-world experiments. Furthermore, successful 3D simulation tests of both decision making stages demonstrate that the developed solution is suitable for complex situations, such as those found in city road traffic environments.

\section{REFERENCES}

Bacha, A., Bauman, C., Faruque, R., Fleming, M., and Terwelp, C. (2008). Odin: Team victortangos entry in the darpa urban challenge. Journal of Field Robotics, 25(8).

Bohren, J., Foote, T., Keller, J., Kushleyev, A., Lee, D., Stewart, A., Vernaza, P., Derenick, J., Spletzer, J., and Satterfield, B. (2008). Little ben: The ben franklin racing teams entry in the 2007 darpa urban challenge. Journal of Field Robotics, 25(9).

Boisse, S., Benenson, R., Bouraoui, L., Parent, M., and Vlacic, L. (2007). Cybernetic transportation systems design and development: Simulation software. In IEEE International Conference on Robotics and Automation ICRA'200\%. Roma, Italy.

Chankong, V. and Haimes, Y.Y. (1983). Multiobjective Decision Making. Elsevier Science Publishing Co., Inc.

DARPA (2006). Urban challenge rules. URL http://www.darpa.mil/grandchallenge/docs/ Urban_Challenge_Rules_121106.pdf.

Furda, A. and Vlacic, L. (2009). Towards increased road safety: Real-time decision making for driverless city vehicles. In 2009 IEEE International Conference on Systems, Man, and Cybernetics. San Antonio, TX, USA.

Kammel, S., Ziegler, J., Pitzer, B., Werling, M., Gindele, T., Jagzent, D., Schroeder, J., Thuy, M., Goebl, M., and Hundelshausen, F.v. (2008). Team annieways autonomous system for the 2007 darpa urban challenge. Journal of Field Robotics, 25(9).
Leonard, J., How, J., Teller, S., Berger, M., Campbell, S., Fiore, G., Fletcher, L., Frazzoli, E., Huang, A., Karaman, S., Koch, O., Kuwata, Y., Moore, D., Olson, E., Peters, S., Teo, J., Truax, R., and Walter, M. (2008). A perception-driven autonomous urban vehicle. Journal of Field Robotics, 25(10), 727-774.

Miller, I., Campbell, M., Huttenlocher, D., and Kline, F.R. (2008). Team cornells skynet: Robust perception and planning in an urban environment. Journal of Field Robotics, 25(8).

Montemerlo, M., Becker, J., Bhat, S., Dahlkamp, H., Dolgov, D., Ettinger, S., and Haehnel, D. (2008). Junior: The stanford entry in the urban challenge. Journal of Field Robotics, 25(9).

Patz, B.J., Papelis, Y., Pillat, R., Stein, G., and Harper, D. (2008). A practical approach to robotic design for the darpa urban challenge. Journal of Field Robotics, $25(8)$.

Rauskolb, F.W., Berger, K., Lipski, C., Magnor, M., Cornelsen, K., Effertz, J., Form, T., Graefe, F., Ohl, S., Schumacher, W., and Wille, J.M. (2008). Caroline: An autonomously driving vehicle for urban environments. Journal of Field Robotics, 25(9).

Stadler, W. (ed.) (1988). Multicriteria optimization in engineering and in the sciences. Plenum Press, New York.

Urmson, C., Anhalt, J., Bagnell, D., Baker, C., Bittner, R., Clark, M.N., Dolan, J., Duggins, D., Galatali, T., Geyer, C., Gittleman, M., Harbaugh, S., Hebert, M., Howard, T.M., Kolski, S., Kelly, A., Likhachev, M., McNaughton, M., Miller, N., Peterson, K., Pilnick, B., Rajkumar, R., Rybski, P., Salesky, B., Seo, Y.W., Singh, S., Snider, J., Stentz, A., Whittaker, W.R., Wolkowicki, Z., and Ziglar, J. (2008). Autonomous driving in urban environments: Boss and the urban challenge. Journal of Field Robotics, $25(8)$.

White, D.J. (1976). Fundamentals of Decision Theory. American Elsevier Publishing Co., Inc.

Yoon, K.P. and Hwang, C.L. (1995). Multiple attribute decision making. SAGE Publications Inc. 